\title{
Prognostic Relevance of a Complete Pathologic Response in Liver Transplantation for Hepatocellular Carcinoma
}

\author{
Michał Grąt, MD, PhD ${ }^{1}$, Marek Krawczyk, MD, PhD ${ }^{1}$, Jan Stypułkowski, MD ${ }^{1}$, Marcin Morawski, MD ${ }^{1}$, \\ Maciej Krasnodębski, MD, $\mathbf{P h D}^{1}$, Michał Wasilewicz, $\mathbf{M D}, \mathbf{P h D}^{2}$, Zbigniew Lewandowski, $\mathbf{P h D}^{3}$, \\ Karolina Grąt, $\mathrm{MD}^{4}$, Waldemar Patkowski, $\mathrm{MD}, \mathrm{PhD}^{1}$, and Krzysztof Zieniewicz, $\mathrm{MD}, \mathrm{PhD}^{1}$ \\ ${ }^{1}$ Department of General, Transplant and Liver Surgery, Medical University of Warsaw, Warsaw, Poland; ${ }^{2}$ Hepatology and \\ Internal Medicine Unit, Department of General, Transplant and Liver Surgery, Medical University of Warsaw, Warsaw, \\ Poland; ${ }^{3}$ Department of Epidemiology and Biostatistics, Medical University of Warsaw, Warsaw, Poland; ${ }^{4}$ Second \\ Department of Clinical Radiology, Medical University of Warsaw, Warsaw, Poland
}

\begin{abstract}
Background. A complete pathologic response (CPR) after neoadjuvant treatment is reported to be associated with an exceptionally low risk of recurrence after liver transplantation for hepatocellular carcinoma (HCC). This study aimed to evaluate the prognostic role of $\mathrm{CPR}$ in liver transplantation for HCC.

Methods. This retrospective cohort study was based on $222 \mathrm{HCC}$ transplant recipients. Incidence of recurrence and survival at 5 years were the primary and secondary outcome measures, respectively. Competing risk analyses were applied to evaluate recurrence incidence and its predictors. Propensity score matching was performed to compare the outcomes for patients after neoadjuvant treatment with and without CPR.

Results. Neoadjuvant treatment was performed for 127 patients, 32 of whom achieved CPR (25.2\%). Comparison of baseline characteristics showed that the patients with CPR were at lowest baseline recurrence risk, followed by treatment-naïve patients and patients without CPR. Adjusted for potential confounders, CPR did not have any significant effects on tumor recurrence. No significant net reclassification improvement was noted after addition of CPR to existing criteria. Neoadjuvant treatment without CPR was associated with increased risk of recurrence in
\end{abstract}

(C) The Author(s) 2019

First Received: 1 June 2019; Published Online: 13 September 2019

M. Grat, $\mathrm{MD}, \mathrm{PhD}$

e-mail: michal.grat@gmail.com subgroups within the Milan criteria $(p=0.016)$, with alpha-fetoprotein concentration (AFP) model not exceeding 2 points $(p=0.021)$ and within the Warsaw criteria $(p=0.007)$ compared with treatment-naïve patients who were at risk similar to those with CPR. The 5-year incidences of recurrence in propensity score-matched patients with and without CPR were respectively $14.0 \%$ and $15.9 \%$ $(p=0.661)$, with corresponding survival rates of $73.2 \%$ and $67.4 \%$, respectively ( $p=0.329$ ).

Conclusions. The findings showed that CPR is not independently associated with long-term outcomes after liver transplantation for HCC.

Liver transplantation remains the optimal treatment for selected patients with hepatocellular carcinoma (HCC). ${ }^{1}$ Considering both transplant utility and survival benefit, selection of HCC patients for transplantation should be aimed at keeping the post-transplantation risk of tumor recurrence within acceptable limits. Historically, this has been accomplished through the use of selection criteria based on morphologic features, namely, number and size of lesions, with the well-known Milan criteria as the benchmark for assessment of patient outcomes during more than over 2 decades. ${ }^{2-4}$ However, selection of patients based solely on gross morphologic features has resulted in inferior post-transplantation outcomes for HCC patients compared with patients who had non-malignant diseases. ${ }^{5}$ The results of numerous observational studies indicate that these drawbacks may be overcome by combining 
morphologic factors with surrogates of aggressive biology, most frequently represented by serum alpha-fetoprotein (AFP) concentration. ${ }^{6-10}$

The response to neoadjuvant treatments before liver transplantation is increasingly recognized as a marker of tumor biology potentially applicable in more precise selection of patients for transplantation. ${ }^{11,12}$ Neoadjuvant treatments including transarterial chemoembolization (TACE), radioembolization, and radiofrequency ablation are commonly used in transplantation centers worldwide, either to decrease the risk of dropout from the waiting list due to tumor progression in patients initially meeting particular selection criteria or to downstage patients beyond them. ${ }^{13-15}$

Although both strategies seem efficient, the direct impact of pre-transplantation neoadjuvant therapies on post-transplantation outcomes remains unclear. ${ }^{16}$ Interestingly, although both modified response evaluation criteria in solid tumors (mRECIST) and European Association for the Study of the Liver criteria are based on assessment of induced necrosis, partial tumor necrosis secondary to neoadjuvant therapies has recently been shown to promote lymphatic HCC dissemination. ${ }^{17,18}$ In contrast, both a complete radiologic tumor response and particularly a complete pathologic response (CPR) are reported to be associated with favorable outcomes. ${ }^{19}$ However, data on the prognostic role of CPR, even referred to as a surrogate for cancer cure, also are inconsistent. ${ }^{20,21}$ Therefore, considering the limited ability to predict tumor response to neoadjuvant treatment and its impact on the risk of tumor recurrence, this study aimed to establish the prognostic relevance of $\mathrm{CPR}$ with respect to $\mathrm{HCC}$ recurrence in patients undergoing liver transplantation.

\section{METHODS}

This retrospective observational study was based on a cohort of 222 consecutive HCC patients after deceased donor liver transplantations performed in the Department of General, Transplant, and Liver Surgery at the Medical University of Warsaw during the period between January 2010 and August 2017. Patients with fibrolamellar HCCs, combined hepatocellular/cholangiocellular carcinomas, and carcinosarcomas were not included in the study.

The study protocol conformed to the ethical guidelines of the 1975 Declaration of Helsinki as reflected in a priori approval by the Medical University of Warsaw human research committee. Due to the retrospective character of the study, informed consent was not required.

A CPR after neoadjuvant therapy, defined as an absence of viable cancer cells on explant pathologic examination, was the primary factor of interest. All histopathologic examinations were performed by pathologists experienced in assessment of hepatobiliary specimens. Neoadjuvant therapies comprised TACE with a mixture of doxorubicin and lipiodol, percutaneous radiofrequency ablation under computed tomography guidance, or a combination of both.

As a routine practice, TACE was performed in cycles of three or two procedures with 4- to 8-week intervals. Patients were individually selected for neoadjuvant treatments during multidisciplinary meetings. In general, patients at presumed high risk of tumor progression beyond Milan criteria were selected for bridging strategy, and those initially beyond Milan criteria were routinely selected for downstaging. Ablative procedures were preferred for patients with solitary lesions up to $3 \mathrm{~cm}$ in size, and TACE was performed for larger solitary lesions or multinodular HCCs.

The cumulative incidence of tumor recurrence during the 5-year post-transplantation period was the primary outcome measure. It was based on an end point of tumor recurrence, with death for non-HCC-related causes considered as a competing risk event. Overall survival, the secondary outcome measure, was calculated from the date of transplantation until the patient's death irrespective of cause and censored 5 years after transplantation or at the last follow-up visit. Data on perioperative management, follow-up protocol, and immunosuppression regimens were provided previously. ${ }^{22,23}$

First, baseline characteristics were compared between patients without neoadjuvant treatments, those who had neoadjuvant treatment without CPR, and those who had neoadjuvant treatment with CPR. The three subgroups were compared with respect to post-transplantation outcomes, both in the entire study cohort and after stratification for the initial risk of tumor recurrence according to Milan criteria, total tumor volume (TTV)/AFP criteria, Metroticket 2.0 criteria, French AFP model, and Warsaw criteria.

The potential prognostic significance of CPR also was evaluated in multivariable analyses adjusted for tumor size, tumor number, last pre-transplant AFP, Metroticket 2.0 model, AFP model, and selected selection criteria. To adjust for differences in baseline risk of tumor recurrence, patients with CPR were additionally matched with those not achieving CPR, and post-transplantation outcomes were compared between the two matched cohorts.

Quantitative and qualitative data were presented respectively as medians with interquartile ranges and as numbers with frequencies. Intergroup comparisons were performed using the Mann-Whitney $U$ test for quantitative variables and the Chi square test or Fisher's exact test for qualitative variables. The cumulative incidence of tumor recurrence and its predictors were analyzed using competing risk regression according to Fine and Gray. ${ }^{24-26}$ 
Overall survival estimates were calculated using the Kaplan-Meier method. The log-rank test was used for comparisons of survival curves. The reverse Kaplan-Meier method was used to establish the median duration of follow-up evaluation. Risk factors for impaired overall survival were analyzed using Cox proportional hazards regression. Predictors of CPR were established using logistic regression models.

The potential relevance of CPR for improved prediction of tumor recurrence and patient mortality was evaluated using the Bayesian information criterion (BIC), which provides a measure for choosing a better prognostic model, with lower values indicating improved efficacy. A difference in BIC between the analyzed model and the baseline model $(\triangle \mathrm{BIC})$ of more than 2 was chosen as the cutoff for omitting the former from further consideration. ${ }^{26}$

Net reclassification improvement (NRI) for adding CPR to the existing selection criteria in prediction of tumor recurrence was established after exclusion of patients with a follow-up period shorter than 2 years. The NRI provides a measure of model predictive performance with respect to improvement of correct reclassification of patients with events and those without events. ${ }^{27}$

Propensity score matching was performed based on the results of logistic regression analyses using the nearest neighbor method in a 1:1 ratio. Hazard ratios (HRs), competing risk regression coefficients $(\beta)$, and NRIs are presented with $95 \%$ confidence intervals (CIs). The level of statistical significance was set at 0.05 . All $p$ values were two-sided. Statistical analyses were computed in STATISTICA version 13.1 (Dell Inc, Tulsa, OK, USA) and R version 3.5.0 (The R Foundation for Statistical Computing, Vienna, Austria).

\section{RESULTS}

Neoadjuvant treatment was performed for 127 (57.2\%) of the 222 patients in the study cohort. This included TACE alone for 88 patients (39.6\%), RFA alone forn 21 patients $(9.5 \%)$, and a combination of TACE and RFA for 18 patients $(8.1 \%)$. Overall, explant pathology showed CPR for $32(25.2 \%)$ of 127 patients after neoadjuvant treatment, including 21 patients after TACE alone (23.9\%), 6 patients after RFA alone (28.6\%), and 5 patients after the TACE and RFA combination $(27.8 \% ; p=0.872)$.

Compared with the patients who received no neoadjuvant treatment, the patients who underwent neoadjuvant treatment without CPR were older $(p=0.026)$ and had a lower model for end-stage liver disease (MELD) score $(p<0.001)$, larger tumors $(p<0.001)$, a greater total tumor volume $(p<0.001)$, a higher AFP model $(p=0.009)$, a higher Metroticket 2.0 model $(p=0.003)$, and a nonsignificantly higher AFP $(p=0.071)$. These patients also were more often beyond the Milan criteria $(p=0.003)$, the AFP model cutoff of 2 points $(p=0.032)$, and the Warsaw criteria $(p=0.019)$.

In contrast, the patients who underwent neoadjuvant treatment with CPR had fewer tumors $(p=0.010)$ and a lower Metroticket 2.0 model $(p<0.001)$. These patients also were more often within both the Milan criteria $(p=0.039)$ and the AFP model cutoff of 2 points $(p=0.042)$, and uniformly within the limits of Metroticket $2.0(p=0.001)$ and the Warsaw criteria $(p=0.001)$ compared with the treatment-naïve patients (Table 1).

During a median follow-up period of 41.3 months, 34 patients experienced tumor recurrence, and 50 patients died. The median time to recurrence was 14.6 months. Overall, the cumulative 5-year incidence of recurrence was $17 \%$ for the patients without neoadjuvant treatment, $28.5 \%$ for the patients who had neoadjuvant treatment without CPR, and $14 \%$ for the patients who had neoadjuvant treatment with CPR $(p=0.157)$. Accordingly, no significant associations between neoadjuvant treatment with CPR $(\exp [\beta]=0.78 ; 95 \%$ CI, $0.23-2.70 ; p=0.700)$ or neoadjuvant treatment without $\mathrm{CPR}(\exp [\beta]=1.79 ; 95 \% \mathrm{CI}$, $0.87-3.70 ; p=0.110)$ and post-transplantation risk of recurrence were identified.

The significant predictors of tumor recurrence were tumor number $(p<0.001)$, AFP $(p=0.001)$, Metroticket $2.0(p<0.001)$, AFP model $(p<0.001)$, TTV/AFP criteria $(p<0.001)$, and Warsaw criteria $(p<0.001)$. After adjustment for their effects in multivariable analyses, a complete response to neoadjuvant treatment was not found to be associated with a decreased risk of tumor recurrence (Table 2). Furthermore, all regression coefficients were positive, pointing toward nonsignificantly increased risk of tumor recurrence after adjustment for these confounders.

Finally, the models incorporating a response to neoadjuvant treatment had a BIC 8.85 to 10.28 higher than the corresponding models without a response to neoadjuvant treatment, pointing toward their worse performance. No benefits regarding improvement of patient selection were observed in terms of NRI when a response to neoadjuvant treatment was added to the AFP model, the Metroticket 2.0 model, the TTV/AFP criteria, the Warsaw criteria, or simply to tumor size, number, and AFP (Table 3).

Considering the general low-risk profile of the patients achieving CPR, a series a subgroup analyses of the patients within the Milan criteria and selected criteria based on a combination of morphologic and biologic factors were performed (Table 4). These showed a uniform nonsignificantly higher incidence of recurrence among the patients who had neoadjuvant treatment with CPR and a significantly increased incidence of recurrence among the patients who underwent neoadjuvant treatment without 
TABLE 1 Baseline characteristics of patients with hepatocellular carcinoma (HCC) who had liver transplantation not preceded by neoadjuvant treatment and those who had prior neoadjuvant treatment with and without a complete pathologic response (CPR)

\begin{tabular}{|c|c|c|c|c|c|}
\hline \multirow[t]{2}{*}{ Factors } & \multirow{2}{*}{ 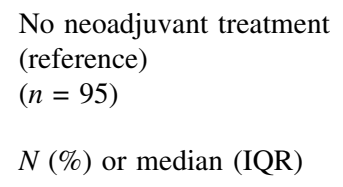 } & \multicolumn{2}{|c|}{$\begin{array}{l}\text { Neoadjuvant treatment without } \\
\text { CPR } \\
(n=95)\end{array}$} & \multicolumn{2}{|c|}{$\begin{array}{l}\text { Neoadjuvant treatment with CPR } \\
(n=32)\end{array}$} \\
\hline & & $n(\%)$ or median (IQR) & $p$ value & $n(\%)$ or median (IQR) & $p$ value \\
\hline Recipient sex (male) & $72(75.8)$ & $71(74.7)$ & $>0.999$ & $25(78.1)$ & $>0.999$ \\
\hline Recipient age (years) & $57(52-61)$ & $58(55-63)$ & 0.026 & $57(56-62)$ & 0.400 \\
\hline MELD (points) & $11.5(9.0-15.0)$ & $9(8-12)$ & $<0.001$ & $9.5(8.0-11.5)$ & 0.011 \\
\hline Hepatitis $\mathrm{C}$ virus & $71(74.7)$ & $68(71.6)$ & 0.744 & $19(59.4)$ & 0.117 \\
\hline Hepatitis B virus & 39 (41.1) & $42(44.2)$ & 0.769 & $18(56.3)$ & 0.154 \\
\hline No. of tumors & $1(1-3)$ & $2(1-3)$ & 0.220 & $1(1-1)$ & 0.010 \\
\hline Size of largest tumor $(\mathrm{cm})$ & $2.5(1.5-3.5)$ & $3.5(2.5-5.0)$ & $<0.001$ & $3.0(2.0-4.5)$ & 0.122 \\
\hline Total tumor volume $\left(\mathrm{cm}^{3}\right)$ & $12.2(2.1-33.5)$ & $32.9(8.7-101.3)$ & $<0.001$ & $14.1(4.2-47.7)$ & 0.324 \\
\hline $\begin{array}{l}\text { Last pre-transplant AFP (ng/ } \\
\text { mL) }\end{array}$ & $10.9(4.6-65.2)$ & $19.8(6.1-158.1)$ & 0.071 & $7.8(4.4-15.7)$ & 0.187 \\
\hline Microvascular invasion & $21(22.3)$ & $28(29.5)$ & 0.320 & - & - \\
\hline Poor tumor differentiation & $11(11.6)$ & $11(11.6)$ & $>0.999$ & - & - \\
\hline 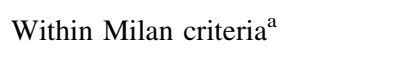 & $65(68.4)$ & $44(46.3)$ & 0.003 & $28(87.5)$ & 0.039 \\
\hline AFP model (points) & $1(0-2)$ & $1(0-3)$ & 0.009 & $1(0-1)$ & 0.367 \\
\hline AFP model $\leq 2$ points & $77(81.1)$ & $63(66.3)$ & 0.032 & $31(96.9)$ & 0.042 \\
\hline Metroticket 2.0 (points) & $1.8(1.4-2.8)$ & $2.4(1.8-3.3)$ & 0.003 & $0.7(0.5-1.0)$ & $<0.001$ \\
\hline Within Metroticket 2.0 criteria $^{\mathrm{b}}$ & $73(76.8)$ & $62(65.3)$ & 0.109 & $32(100.0)$ & 0.001 \\
\hline Within TTV/AFP criteria ${ }^{c}$ & $81(85.3)$ & $73(77.7)$ & 0.195 & $31(96.9)$ & 0.113 \\
\hline Within Warsaw criteria ${ }^{\mathrm{d}}$ & $73(76.8)$ & $57(60.0)$ & 0.019 & $32(100.0)$ & 0.001 \\
\hline Donor age (years) & $50(42-60)$ & $52(39-61)$ & 0.570 & $57(42-63)$ & 0.199 \\
\hline Donor sex (male) & $63(66.3)$ & $55(57.9)$ & 0.295 & $17(53.1)$ & 0.207 \\
\hline
\end{tabular}

$I Q R$ interquartile range, $M E L D$ model for end-stage liver disease, $A F P$ alpha-fetoprotein, $T T V$ total tumor volume

${ }^{a}$ Milan criteria: 1 tumor $\leq 5 \mathrm{~cm}$ or $2-3$ tumors $\leq 3 \mathrm{~cm}$

${ }^{\mathrm{b}}$ Metroticket 2.0 criteria: sum of the size of the largest tumor in centimeters $\leq 4$ and AFP $\leq 1000 \mathrm{ng} / \mathrm{mL}$ or sum of the size of the largest tumor in centimeters $\leq 5$ and AFP $\leq 400 \mathrm{ng} / \mathrm{mL}$ or sum of the size of the largest tumor in centimeters $\leq 7 \mathrm{and} \mathrm{AFP} \leq 200 \mathrm{ng} / \mathrm{mL}$

${ }^{\mathrm{c}}$ TTV/AFP criteria: $\mathrm{TTV} \leq 115 \mathrm{~cm}^{3}$ and AFP $\leq 400 \mathrm{ng} / \mathrm{mL}$

${ }^{\mathrm{d}}$ Warsaw criteria: within Milan criteria or within either University of California, San Francisco criteria or up-to-7 criteria with AFP $\leq 100 \mathrm{ng} /$ $\mathrm{mL}$

CPR within the Milan criteria $(p=0.016)$, the Warsaw criteria $(p=0.007)$, or the AFP model not exceeding 2 points $(p=0.021)$.

The overall survival rate was $79.5 \%$ at 5 years for the patients without neoadjuvant treatment, $66.1 \%$ for those who underwent neoadjuvant treatment without CPR, and $73.2 \%$ for those who underwent neoadjuvant treatment with CPR $(p=0.363)$. Accordingly, neither the presence (HR, 1.21; 95\% CI, 0.50-2.89; $p=0.938$ ) nor the absence (HR, $1.55 ; 95 \%$ CI, 0.85-2.84; $p=0.249$ ) of CPR was a significant prognostic factor for overall survival. The AFP model $(p=0.022)$, the TTV/AFP criteria $(p=0.013)$, and the Warsaw criteria $(p=0.001)$ were significantly associated with overall survival, whereas the Metroticket 2.0 model did not reach significance $(p=0.109)$. After adjustment for their effects, CPR was not associated with the risk of tumor recurrence, with the absolute HRs ranging from 1.32 to 1.63 (Table 5).

Comparison of BIC values also did not support inclusion of a response to neoadjuvant treatment with the existing criteria in terms of predicting overall survival. For the patients within Milan criteria, the AFP model not exceeding 2 points, the Metroticket 2.0 criteria, the TTV/AFP criteria, and the Warsaw criteria including both CPR and lack of CPR were associated with nonsignificantly lower overall survival rates (Table 4).

The patients achieving CPR were matched in a 1:1 ratio with the patients who did not achieve CPR using a propensity score based on tumor number, tumor size, and AFP. In this matched cohort, the 5-year cumulative 
TABLE 2 Results of multivariable competing risk regression analyses of risk factors for tumor recurrence including morphologic variables combined with AFP having no response to neoadjuvant treatment and corresponding models with response to neoadjuvant treatment

\begin{tabular}{llllll}
\hline Models & $\operatorname{Exp}(\beta)$ & $95 \%$ CI for $\exp (\beta)$ & $p$ Value & BIC & $\Delta$ BIC $^{\mathrm{a}}$ \\
\hline Model 1A & & & & 331.86 & 10.19 \\
No. of tumors & 1.23 & $1.10-1.37$ & $<0.001$ & & \\
Size of largest tumor & 1.01 & $0.99-1.03$ & 0.240 & & \\
AFP & 1.85 & $1.28-2.67$ & 0.001 & &
\end{tabular}

Model 1B

No. of tumors

1.23

$1.10-1.38$

342.05

Size of largest tumor

0.99-1.03

$<0.001$

1.01

$1.28-2.74$

0.350

1.87

0.001

Neoadjuvant treatment

No

Reference

Yes, with no CPR

$\begin{array}{ll}1.33 & 0.61-2.90 \\ 1.34 & 0.37-4.85\end{array}$

0.470

Yes, with CPR

1.34

$0.37-4.85$

0.660

1.84

$1.40-2.41$

324.49

9.56

Metroticket 2.0

Model 2B

Metroticket 2.0

Neoadjuvant treatment

No

Yes, with no CPR

Yes, with CPR

1.88

$1.37-2.57$

$<0.001$

$<0.001$

Reference

1.27

$0.58-2.75$

0.550

2.10

$0.54-8.19$

0.280

Model 3A

1.55

$1.30-1.84$

$<0.001$

Model 3B

AFP model

1.52

$1.25-1.85$

$<0.001$

Reference

$\begin{array}{lll}1.30 & 0.59-2.88 & 0.510 \\ 1.03 & 0.31-3.38 & 0.960\end{array}$

5.82

$2.96-11.40$

326.78

8.85

Beyond TTV/AFP criteria

Model 4B

Beyond TTV/AFP criteria

Neoadjuvant treatment:

No

Yes, with no CPR

Yes, with CPR

5.52

2.73-11.16

$<0.001$

335.63

Reference

$\begin{array}{lll}1.65 & 0.78-3.48 & 0.190\end{array}$

1.09

$0.33-3.58$

0.880

Model 5A

Beyond Warsaw criteria

4.26

$2.19-8.28$

$<0.001$

4.05

1.81-9.09

0.001

Beyond Warsaw criteria

Neoadjuvant treatment

No

Reference

Yes, with no CPR

1.31

$0.58-2.95$

0.520

1.21

$0.35-4.13$

0.760

Competing risk regression coefficients were calculated for 1 tumor increase for the number of tumors, $1-\mathrm{mm}$ increase for tumor size, $1-\log _{10}(\mathrm{ng} / \mathrm{mL})$ increase for AFP, 1-point increase for Metroticket 2.0 model and AFP model

$A F P$ alpha-fetoprotein, $\beta$ competing risk regression coefficient, $C I$ confidence interval, BIC Bayesian information criterion, $C P R$ complete pathologic response, $T T V$ total tumor volume

${ }^{\mathrm{a}} \Delta \mathrm{BIC}=\mathrm{BIC}($ model $\mathrm{B})-\mathrm{BIC}(\operatorname{model} \mathrm{A})$ 
TABLE 3 Net reclassification improvement (NRI) analyses of adding neoadjuvant treatment response to tumor burden and alpha-fetoprotein in selection of hepatocellular patients for liver transplantation with respect to tumor recurrence prediction

\begin{tabular}{|c|c|c|c|c|}
\hline \multirow[t]{2}{*}{ Baseline model } & \multicolumn{4}{|c|}{ NRI for modification of baseline model by adding response to neoadjuvant treatment } \\
\hline & NRI & $95 \%$ CI for NRI & $Z$ & $p$ value \\
\hline $\begin{array}{l}\text { Model } 1 \\
\text { Tumor number } \\
\text { Tumor size } \\
\text { Alpha-fetoprotein }\end{array}$ & -0.018 & -0.067 to 0.032 & -0.055 & 0.478 \\
\hline $\begin{array}{l}\text { Model } 2 \\
\text { AFP model }\end{array}$ & 0.056 & -0.021 to 0.133 & 0.115 & 0.546 \\
\hline $\begin{array}{l}\text { Model } 3 \\
\text { Metroticket } 2.0 \text { model }\end{array}$ & -0.005 & -0.061 to 0.052 & -0.013 & 0.495 \\
\hline $\begin{array}{l}\text { Model } 4 \\
\text { TTV/AFP }_{\text {criteria }}{ }^{\mathrm{a}}\end{array}$ & $--^{\mathrm{a}}$ & - & - & - \\
\hline $\begin{array}{l}\text { Model } 5 \\
\text { Warsaw criteria }\end{array}$ & 0.038 & -0.079 to 0.155 & 0.051 & 0.520 \\
\hline
\end{tabular}

$C I$ confidence interval, AFP alpha-fetoprotein, TTV total tumor volume

${ }^{a}$ Response to neoadjuvant treatment added to TTV/AFP criteria did not reclassify any patient

TABLE 4 Cumulative incidences of recurrence and Kaplan-Meier overall survival estimates 5 years after liver transplantation for hepatocellular carcinoma for patients in selected low-risk subgroups based on response to neoadjuvant treatment

\begin{tabular}{|c|c|c|c|c|c|c|c|}
\hline \multirow[t]{2}{*}{ Outcome measure } & \multirow[t]{2}{*}{ Subgroup } & \multirow{2}{*}{$\begin{array}{l}\text { No neoadjuvant } \\
\text { treatment } \\
\text { (reference) } \\
\text { 5-Year estimate } \\
(\%)\end{array}$} & \multicolumn{2}{|c|}{$\begin{array}{l}\text { Neoadjuvant treatment } \\
\text { without CPR }\end{array}$} & \multicolumn{2}{|c|}{$\begin{array}{l}\text { Neoadjuvant treatment } \\
\text { with CPR }\end{array}$} & \multirow[t]{2}{*}{$\begin{array}{l}\text { Overall } \\
p \text { value }\end{array}$} \\
\hline & & & $\begin{array}{l}\text { 5-Year } \\
\text { estimate } \\
(\%)\end{array}$ & $p$ value $^{\mathrm{a}}$ & $\begin{array}{l}\text { 5-Year } \\
\text { estimate } \\
(\%)\end{array}$ & $p$ value $^{\mathrm{a}}$ & \\
\hline $\begin{array}{l}\text { Incidence of } \\
\text { recurrence }\end{array}$ & Within Milan criteria & 5.0 & 22.2 & 0.016 & 13.3 & 0.290 & 0.027 \\
\hline $\begin{array}{l}\text { Incidence of } \\
\text { recurrence }\end{array}$ & AFP model $\leq 2$ & 7.4 & 23.8 & 0.021 & 10.4 & 0.740 & 0.033 \\
\hline $\begin{array}{l}\text { Incidence of } \\
\text { recurrence }\end{array}$ & $\begin{array}{l}\text { Within Metroticket } 2.0 \\
\text { criteria }\end{array}$ & 9.2 & 21.1 & 0.110 & 14.0 & 0.580 & 0.257 \\
\hline $\begin{array}{l}\text { Incidence of } \\
\text { recurrence }\end{array}$ & Within TTV/AFP criteria & 9.7 & 23.6 & 0.120 & 10.4 & 0.940 & 0.197 \\
\hline $\begin{array}{l}\text { Incidence of } \\
\text { recurrence }\end{array}$ & Within Warsaw criteria & 4.5 & 25.4 & 0.007 & 14.0 & 0.130 & 0.008 \\
\hline Overall survival & Within Milan criteria & 83.6 & 78.5 & 0.903 & 68.0 & 0.249 & 0.634 \\
\hline Overall survival & AFP model $\leq 2$ & 82.9 & 70.6 & 0.263 & 72.1 & 0.359 & 0.541 \\
\hline Overall survival & $\begin{array}{l}\text { Within Metroticket } 2.0 \\
\text { criteria }\end{array}$ & 82.0 & 70.2 & 0.298 & 73.2 & 0.474 & 0.602 \\
\hline Overall survival & Within TTV/AFP criteria & 82.4 & 69.3 & 0.221 & 72.1 & 0.383 & 0.507 \\
\hline Overall survival & Within Warsaw criteria & 84.0 & 75.0 & 0.655 & 73.2 & 0.395 & 0.837 \\
\hline
\end{tabular}

$C P R$ complete pathologic response, $A F P$ alpha-fetoprotein, $T T V$ total tumor volume

${ }^{\mathrm{a}}$ Relative to patients without neoadjuvant treatment

incidence of recurrence was $14 \%$ for the patients who had neoadjuvant treatment and achieved CPR compared with $15.9 \%$ for those who did not achieve CPR $(p=0.661$;
Fig. 1a). The corresponding incidence rates for non-HCCrelated mortality adjusted for the competing risk of recurrence were $23.7 \%$ and $26.0 \%$, respectively $(p=0.477$, 
TABLE 5 Results from multivariable analyses of risk factors for worse overall survival including morphologic variables combined with AFP having no response to neoadjuvant treatment and corresponding models with response to neoadjuvant treatment

\begin{tabular}{|c|c|c|c|c|c|}
\hline Models & $\mathrm{HR}^{\mathrm{a}}$ & $95 \% \mathrm{CI}$ for $\mathrm{HR}$ & $p$ value & $\mathrm{BIC}$ & $\Delta \mathrm{BIC}^{\mathrm{b}}$ \\
\hline Model 1A & & & & 514.67 & 6.09 \\
\hline Metroticket 2.0 & 1.19 & $0.96-1.47$ & 0.109 & & \\
\hline Model 1B & & & & 520.76 & \\
\hline Metroticket 2.0 & 1.20 & $0.94-1.52$ & 0.144 & & \\
\hline \multicolumn{6}{|l|}{ Neoadjuvant treatment } \\
\hline No & Reference & & & & \\
\hline Yes, with no CPR & 1.44 & $0.77-2.66$ & 0.676 & & \\
\hline Yes, with CPR & 1.56 & $0.60-4.01$ & 0.573 & & \\
\hline Model 2A & & & & 512.36 & 6.48 \\
\hline AFP model & 1.19 & $1.03-1.38$ & 0.022 & & \\
\hline Model 2B & & & & 518.84 & \\
\hline AFP model & 1.18 & $1.01-1.38$ & 0.039 & & \\
\hline \multicolumn{6}{|l|}{ Neoadjuvant treatment } \\
\hline No & Reference & & & & \\
\hline Yes, with no CPR & 1.42 & $0.77-2.63$ & 0.493 & & \\
\hline Yes, with CPR & 1.32 & $0.55-3.19$ & 0.805 & & \\
\hline Model 3A & & & & 511.65 & 6.10 \\
\hline Beyond TTV/AFP criteria & 2.24 & $1.19-4.23$ & 0.013 & & \\
\hline Model 3B & & & & 517.75 & \\
\hline Beyond TTV/AFP criteria & 2.19 & $1.15-4.19$ & 0.017 & & \\
\hline \multicolumn{6}{|l|}{ Neoadjuvant treatment } \\
\hline No & Reference & & & & \\
\hline Yes, with no CPR & 1.49 & $0.81-2.74$ & 0.403 & & \\
\hline Yes, with CPR & 1.34 & $0.55-3.23$ & 0.826 & & \\
\hline Model 4A & & & & 507.73 & 6.29 \\
\hline Beyond Warsaw criteria & 2.51 & $1.44-4.40$ & 0.001 & & \\
\hline Model 4B & & & & 514.02 & \\
\hline Beyond Warsaw criteria & 2.59 & $1.41-4.75$ & 0.002 & & \\
\hline \multicolumn{6}{|l|}{ Neoadjuvant treatment } \\
\hline No & Reference & & & & \\
\hline Yes, with no CPR & 1.35 & $0.73-2.50$ & 0.858 & & \\
\hline Yes, with CPR & 1.63 & $0.66-4.05$ & 0.440 & & \\
\hline
\end{tabular}

$A F P$ alpha-fetoprotein, $H R$ hazard ratio, $C I$ confidence interval, $B I C$ Bayesian information criterion, $C P R$ complete pathological response, TTV total tumor volume

${ }^{\mathrm{a}}$ Hazard ratios were calculated for 1 point increase for the Metroticket 2.0 model and the AFP model

${ }^{\mathrm{a}} \Delta \mathrm{BIC}=\mathrm{BIC}(\operatorname{model} \mathrm{B})-\mathrm{BIC}(\operatorname{model} \mathrm{A})$
Fig. 1a). The overall survival rate was $73.2 \%$ for the patients who achieved CPR and $67.4 \%$ for the patients without CPR ( $p=0.329$, Fig. 1b).

\section{DISCUSSION}

A CPR to neoadjuvant treatment is commonly reported as an extremely favorable factor associated with minimal risk of HCC recurrence after liver transplantation. ${ }^{19,20,28}$ The results of this study indicate that the favorable outcomes for HCC patients who achieve CPR after neoadjuvant treatment are due to their favorable baseline profile of recurrence risk rather than the prognostic impact of the response. According to performed analyses adjusted for the confounding effects of differences in baseline characteristics, assessment of CPR to neoadjuvant treatment does not improve the capability of selection criteria combining morphologic features with AFP to predict tumor recurrence.

In general, CPR was associated with post-transplantation outcomes similar to those for the neoadjuvant treatment-naïve patients and nonsignificantly worse than for the patients without CPR. Irrespective of statistical significance, such absolute differences appear to be in line 

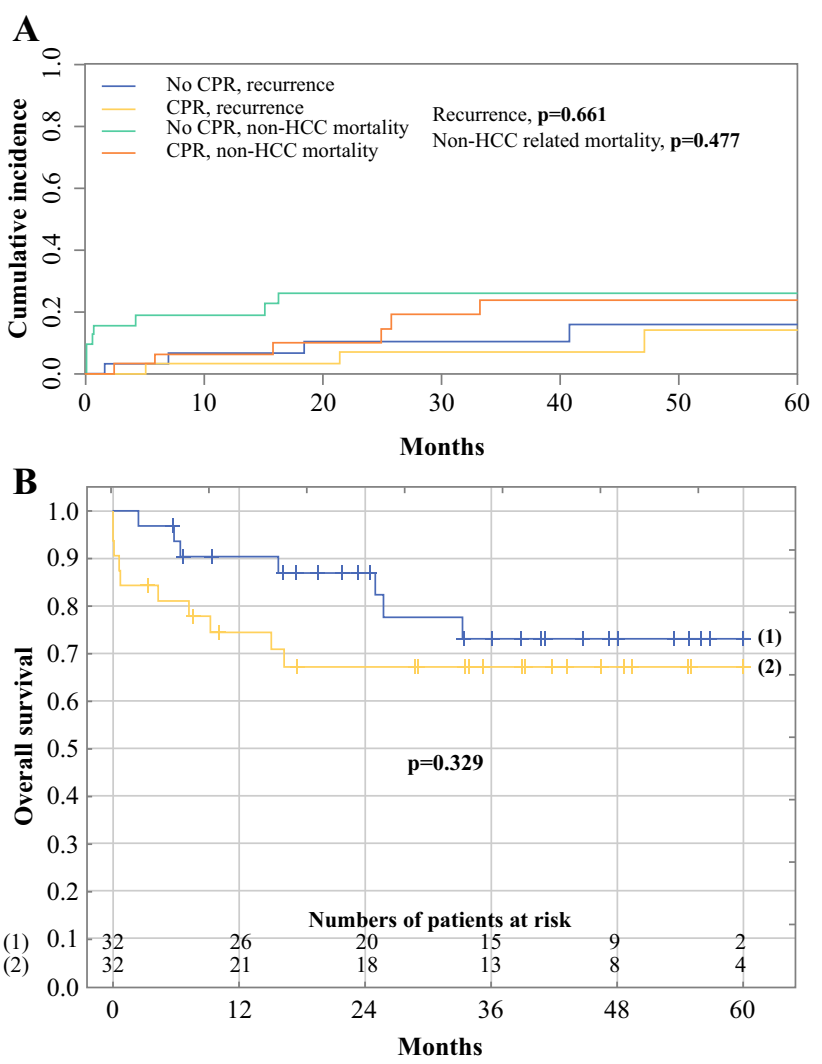

FIG. 1 Comparison of propensity score-matched patients with and without a complete pathologic response (CPR) after neoadjuvant therapy with respect to a competing risk-adjusted cumulative incidence of tumor recurrence and non-hepatocellular carcinoma (HCC)-related mortality, and b overall survival after liver transplantation (blue line-patients with CPR; yellow line-patients without CPR)

with differences in patients' baseline risk factors for tumor recurrence, with the lowest risk expected for patients with CPR followed by treatment-naïve patients and those without CPR after neoadjuvant therapy. Notably, the favorable characteristics of patients who achieve CPR are uniformly reported in other studies, with predictors of response often being well-established predictors of tumor recurrence..$^{20,28,29}$

Notably, none of the performed multivariable analyses pointed toward even a nonsignificant positive effect of CPR in terms of decreased risk for tumor recurrence. After adjustment for the confounding effects of different baseline characteristics through propensity score matching, the patients who achieved and those did not achieve CPR were observed to have almost identical post-transplantation outcomes. Nevertheless, the results do not undermine the rationale for performing neoadjuvant therapy to achieve CPR because it is associated with favorable changes in other risk factors and thus may limit the risk of tumor recurrence. For instance, previous studies have shown that lowering the AFP level after neoadjuvant treatment remarkably limits the risk of post-transplantation recurrence and obviously may enable liver transplantation if values drop below the cutoff for particular criteria. ${ }^{30-32}$

Although the lack of a prognostic role for CPR to neoadjuvant treatment was a rather unexpected finding, a previous study by Kang et al. ${ }^{21}$ showed that patients who underwent either liver resection or transplantation after neoadjuvant treatment had a significantly higher risk of recurrence than low-risk treatment-naïve patients despite achievement of CPR. Importantly, low-risk control groups in that previous study comprised patients with single tumors smaller than $2 \mathrm{~cm}$ eligible for liver resection and up to two tumors smaller than $2 \mathrm{~cm}$ eligible for liver transplantation. In that setting, comparison of baseline characteristics showed that the patients with CPR were in fact at increased baseline risk of tumor recurrence, as indicated by a higher AFP level and a larger tumor size.

The results of this study confirm that a combination of morphologic features with AFP enables selection of HCC patients for liver transplantation, in line with numerous previous studies. ${ }^{6-10}$ The obtained results show that the addition of another biologic criterion represented by a complete response to neoadjuvant treatment not only does not improve the predictive efficacy of those models, but also is associated with their worse performance, a finding reported for the first time in the literature. Notably, none of the previous studies demonstrated that predictive ability improved when a response to neoadjuvant treatment was added to the existing morphologic and biologic criteria. Importantly, the last pre-transplantation patient characteristics were used for patient matching and adjustment of the effects from CPR because these are particularly associated with both the response to treatment and the risk of recurrence. ${ }^{20,30-32}$

Interestingly, neoadjuvant treatment without CPR was associated with significantly higher recurrence rates for several low-risk groups of patients. These included patients within the Milan criteria, those with an AFP model not exceeding 2 points, and those within the Warsaw criteria. The unexpectedly high rates of tumor recurrence for these generally low-risk patients receiving neoadjuvant treatment correspond to the results of a recent large-scale study by the U.S. Multicenter HCC Transplant Consortium, in which neoadjuvant treatment without CPR was independently associated with higher risk of recurrence. ${ }^{30}$

Because the current study aimed to investigate the prognostic effect of CPR, the results are insufficient to support the conclusion that neoadjuvant therapy may potentially increase the risk of post-transplantation recurrence for low-risk HCC patients. The potential reasons for such an association were, however, provided in a recent study by Xu et al. ${ }^{18}$, in which neoadjuvant therapy-induced necrosis was associated with increased lymphangiogenesis 
and increased risk of post-transplantation lymphatic metastases. Therefore, this is another report on the potential negative effect of neoadjuvant therapy and may be considered as an argument for more cautious selection of low-risk patients for pre-transplantation bridging.

Although the results of the current study contradict the prognostic relevance of $\mathrm{CPR}$ and the rationale for its evaluation during pre-transplantation assessment, they do not undermine the role of general radiologic assessment of response to neoadjuvant treatment before liver transplantation. Progression of the disease during the pretransplantation period is consistently reported as a negative prognostic factor for post-transplantation recurrence, and the current study did not reevaluate its prognostic impact. ${ }^{19,33-35}$ On the contrary, assessments of the optimal method for predicting CPR seem unnecessary in the context of the presented results.

The current study had several limitations. First, its retrospective character was associated with all the drawbacks inherent to retrospective studies. Second, the study did not include any data on tumor progression in the pretransplantation period. However, it aimed to evaluate the prognostic significance of CPR and not a response to treatment in general. Furthermore, there were no data on radiologic assessment of response to treatment, yet pathologic evaluation is the gold standard for evaluating a complete response to treatment. ${ }^{17,36}$ Nevertheless, the results indicating that CPR lacks prognostic significance should not be extrapolated to mean that radiologic assessment of a treatment response lacks prognostic significance.

In conclusion, CPR after neoadjuvant treatment does not have prognostic significance in liver transplantation for HCC when adjusted for the effects of differences in baseline characteristics. Patients with low-risk HCCs should cautiously be selected for bridging therapies due to the potential increase of the risk for post-transplantation tumor recurrence.

ACKNOWLEDGEMENT Michał Grat was supported by the Foundation for Polish Science (FNP) with a START stipend (START 032.2018). No other funding is associated with the presented research.

\section{CONFLICT OF INTEREST There are no conflict of interest.}

OPEN ACCESS This article is distributed under the terms of the Creative Commons Attribution 4.0 International License (http://crea tivecommons.org/licenses/by/4.0/), which permits unrestricted use, distribution, and reproduction in any medium, provided you give appropriate credit to the original author(s) and the source, provide a link to the Creative Commons license, and indicate if changes were made.

\section{REFERENCES}

1. Lai Q, Vitale A, Iesari S, et al. Intention-to-treat survival benefit of liver transplantation in patients with hepatocellular cancer. Hepatology. 2017;66:1910-19.

2. Mazzaferro V, Battiston C, Sposito C. Pro (with caution): extended oncologic indications in liver transplantation. Liver Transplant. 2018;24:98-103.

3. Costentin CE, Bababekov YJ, Zhu AX, Yeh H. Is it time to reconsider the Milan criteria for selecting patients with hepatocellular carcinoma for deceased-donor liver transplantation? Hepatology. 2018. https://doi.org/10.1002/hep.30278.

4. Clavien PA, Lesurtel M, Bossuyt PM, Gores GJ, Langer B, Perrier A; OLT for HCC Consensus Group. Recommendations for liver transplantation for hepatocellular carcinoma: an international consensus conference report. Lancet Oncol. 2012;13: e11-22.

5. Grąt M, Stypułkowski J, Patkowski W, et al. Challenging the principle of utility as a barrier for wider use of liver transplantation for hepatocellular cancer. Ann Surg Oncol. 2017;24:3188-95.

6. Halazun KJ, Tabrizian P, Najjar M, et al. Is it time to abandon the milan criteria? Results of a bicoastal U.S. collaboration to redefine hepatocellular carcinoma liver transplantation selection policies. Ann Surg. 2018;268:690-9.

7. Duvoux C, Roudot-Thoraval F, Decaens T, et al. Liver transplantation for hepatocellular carcinoma: a model including $\alpha$ fetoprotein improves the performance of Milan criteria. Gastroenterology. 2012;143:986-94.

8. Grąt M, Wronka KM, Stypułkowski J, et al. The Warsaw proposal for the use of extended selection criteria in liver transplantation for hepatocellular cancer. Ann Surg Oncol. 2017;24:526-34.

9. Mazzaferro V, Sposito C, Zhou J, et al. Metroticket 2.0 model for analysis of competing risks of death after liver transplantation for hepatocellular carcinoma. Gastroenterology. 2018;154:128-39.

10. Toso C, Meeberg G, Hernandez-Alejandro R, et al. Total tumor volume and alpha-fetoprotein for selection of transplant candidates with hepatocellular carcinoma: a prospective validation. Hepatology. 2015;62:158-65.

11. Lai Q, Nicolini D, Inostroza Nunez M, et al. A novel prognostic index in patients with hepatocellular cancer waiting for liver transplantation: Time-Radiological-Response-Alpha-fetoproteinINflammation (TRAIN) score. Ann Surg. 2016;264:787-96.

12. Firl DJ, Kimura S, McVey J, et al. Reframing the approach to patients with hepatocellular carcinoma: longitudinal assessment with HALTHCC improves ablate and wait strategy. Hepatology. 2018. https://doi.org/10.1002/hep.29907.

13. Kim Y, Stahl CC, Makramalla A, et al. Downstaging therapy followed by liver transplantation for hepatocellular carcinoma beyond Milan criteria. Surgery. 2017;162:1250-8.

14. Toso C, Meeberg G, Andres A, et al. Downstaging prior to liver transplantation for hepatocellular carcinoma: advisable but at the price of an increased risk of cancer recurrence: a retrospective study. Transpl Int. 2018. https://doi.org/10.1111/tri.13337.

15. Pinna AD, Yang T, Mazzaferro V, et al. Liver transplantation and hepatic resection can achieve cure for hepatocellular carcinoma. Ann Surg. 2018. https://doi.org/10.1097/SLA.0000000000002889.

16. Kulik L, Heimbach JK, Zaiem F, et al. Therapies for patients with hepatocellular carcinoma awaiting liver transplantation: a systematic review and meta-analysis. Hepatology. 2018;67:381-400.

17. Dioguardi Burgio M, Ronot M, Bruno O, et al. Correlation of tumor response on computed tomography with pathological necrosis in hepatocellular carcinoma treated by chemoembolization before liver transplantation. Liver Transplant. 2016;22:1491-500. 
18. Xu M, Doyle MM, Banan B, et al. Neoadjuvant locoregional therapy and recurrent hepatocellular carcinoma after liver transplantation. J Am Coll Surg. 2017;225:28-40.

19. Lee DD, Samoylova M, Mehta N, et al. mRECIST provides insight into tumor biology for patients with hepatocellular carcinoma awaiting liver transplantation. Liver Transplant. 2018. h ttps://doi.org/10.1002/lt.25333

20. Agopian VG, Morshedi MM, McWilliams J, et al. Complete pathologic response to pretransplant locoregional therapy for hepatocellular carcinoma defines cancer cure after liver transplantation: analysis of 501 consecutively treated patients. Ann Surg. 2015;262:536-45.

21. Kang WH, Hwang S, Song GW, et al. Prognostic effect of transarterial chemoembolization-induced complete pathological response in patients undergoing liver resection and transplantation for hepatocellular carcinoma. Liver Transplant. 2017;23: 781-90.

22. Grat M, Kornasiewicz O, Lewandowski Z, et al. The impact of surgical technique on the results of liver transplantation in patients with hepatocellular carcinoma. Ann Transplant. 2013;18: $448-59$.

23. Krawczyk M, Grąt M, Barski K, et al. 1000 Liver transplantations at the Department of General, Transplant and Liver Surgery, Medical University of Warsaw: analysis of indications and results. Pol Przegl Chir. 2012;84:304-12.

24. Fine JP, Gray RJ. A proportional hazards model for the subdistribution of a competing risk. J Am Stat Assoc. 1999;94:496-509.

25. Scrucca L, Santucci A, Aversa F. Competing risk analysis using R: an easy guide for clinicians. Bone Marrow Transplant. 2007;40:381-7.

26. Scrucca L, Santucci A, Aversa F. Regression modeling of competing risk using R: an in-depth guide for clinicians. Bone Marrow Transplant. 2010;45:1388-95.

27. Leening MJ, Vedder MM, Witteman JC, Pencina MJ, Steyerberg EW. Net reclassification improvement: computation, interpretation, and controversies: a literature review and clinician's guide. Ann Intern Med. 2014;160:122-31.

28. Allard MA, Sebagh M, Ruiz A, et al. Does pathological response after transarterial chemoembolization for hepatocellular carcinoma in cirrhotic patients with cirrhosis predict outcome after liver resection or transplantation? J Hepatol. 2015;63:83-92.

29. Kornberg A, Witt U, Matevossian E, et al. Extended postinterventional tumor necrosis: implication for outcome in liver transplant patients with advanced HCC. PLOS ONE. 2013;8: e53960.

30. Agopian VG, Harlander-Locke MP, Ruiz RM, et al. Impact of pretransplant bridging locoregional therapy for patients with hepatocellular carcinoma within Milan criteria undergoing liver transplantation: analysis of 3601 patients from the U.S. Multicenter HCC Transplant Consortium. Ann Surg. 2017;266:525-35.

31. Merani S, Majno P, Kneteman NM, et al. The impact of waiting list alpha-fetoprotein changes on the outcome of liver transplant for hepatocellular carcinoma. J Hepatol. 2011;55:814-9.

32. Grąt M, Krasnodębski M, Patkowski W, et al. Relevance of pretransplant $\alpha$-fetoprotein dynamics in liver transplantation for hepatocellular cancer. Ann Transplant. 2016;21:115-24.

33. Sandow TA, Arndt SE, Albar AA, et al. Assessment of response to transcatheter arterial chemoembolization with doxorubicineluting microspheres: tumor biology and hepatocellular carcinoma recurrence in a 5-year transplant cohort. Radiology. 2018;286:1072-83.

34. Nicolini D, Agostini A, Montalti R, et al. Radiological response and inflammation scores predict tumour recurrence in patients treated with transarterial chemoembolization before liver transplantation. World J Gastroenterol. 2017;23:3690-701.

35. Morris PD, Laurence JM, Yeo D, et al. Can response to locoregional therapy help predict longterm survival after liver transplantation for hepatocellular carcinoma? A systematic review. Liver Transplant. 2017;23:375-85.

36. Gordic S, Corcuera-Solano I, Stueck A, et al. Evaluation of HCC response to locoregional therapy: validation of MRI-based response criteria versus explant pathology. J Hepatol. 2017;67: 1213-21.

Publisher's Note Springer Nature remains neutral with regard to jurisdictional claims in published maps and institutional affiliations. 